data were analysed through content analysis by doing category, code and prepare theme.

Result and discussion After receiving family therapy and education, parents are able to gain a good knowledge about their child's condition. They also learned how to make their child able in doing functional activities such as self care, study and play activities by using structured home environment with daily visual schedule, some sorts of sensory stimulation, visual timing, using sign of toilet, dining, kitchen etc. Through doing these activities with family members, their children are able to learn about timing of doing things, sequence and steps of doing activities.

Conclusion Parents find it helpful to create a friendly environment for their children at home by family therapy and education.

\section{DEVELOPMENT OUTCOME OF EXTREME PRETERM INFANTS AT 2 YEARS; HAS THE OUTCOME CHANGED OVER LAST FEW YEARS?}

doi:10.1136/archdischild-2012-302724.1249

0 Narayan. Paediatrics, Princess Royal University Hospital, Orpington, UK

Background and Aims Extremely low and very low gestational age (ELGA and VLGA) constitutes a risk factor for development even in absence of cerebral damage, as an immature central nervous system is exposed to invasive and inadequate stimulation. Different developmental trajectories emerged in relation to GA, with poorer developmental outcomes and higher rates of impairment in ELGAs and few mild impairments in VLGAs.

Method A retrospective Audit was done. All new-borns at Princess Royal University Hospital, Orpington, UK $(<30+0)$ between Jan 2008-Dec 2009 were included.

The parameters were as follows:

Gender

Gestation

Significant Neonatal intervention

Referred by hospital for dev asses or not

Assessed by community Paediatrcians or not

Assessed at 2 years or not $(\mathrm{H} / \mathrm{C})$

Referred to portage services or not if delay

Development delay if any

Outcome documented or not

Results Total no of cases:

\section{8: 26}

2009: 23

Total: 49

47 eligible (as 2 had died)

10 (from out of borough or moved out so not followed up) so 37 eligible

29/37 (78\%) followed up

\section{Delay}

\author{
Severe $6(21 \%)$ \\ Mild 9 (31\%) \\ No delay $14(48 \%)$
}

These results are very similar to EPICure studies done in 2006.

Conclusions Children born prematurely still have a higher chance of physical impairment, 3-5 times higher rate of cognitive deficits at 6 years, 2-3 times higher risk of psychiatric diagnosis at 11-12 years. (EPICure).

To have the best possible outcome of these children one should follow them in a multidisciplinary team possibly in an integrated care pathway.

\section{DELUSIONAL IDEATION IN YOUNG ADULTHOOD IS ASSOCIATED WITH GREY AND WHITE MATTER ALTERATIONS IN ADOLESCENCE}

doi:10.1136/archdischild-2012-302724.1250

AK Kalpakidou, MP Allin, M Walshe, KW Nam, PK McGuire, L Rifkin, RM Murray, C Nosarti. Department of Psychosis Studies, Institute of Psychiatry, King's College London, London, UK

Background and Aims Several studies have described an association between very preterm birth and psychiatric problems later in life. We aimed to investigate whether young adults who were born very preterm (VPT) (<33 gestational weeks) are at increased risk of experiencing non-clinical psychotic symptoms compared to controls (e.g., delusional ideation) and whether such symptoms are associated with altered brain maturation.

Methods Sixty-four VPT born individuals and 39 controls (mean age 20 years) completed the Peters' Delusional Inventory, which measures psychosis proneness in the general population. Structural MRI data collected at age 15 years were used to investigated possible anatomical correlates of psychosis proneness, by subdividing the sample according to high (>=8; VPT: $40.6 \%$, controls: $48.7 \%$ ) and low $(<8)$ PDI scores.

Results The groups did not differ in PDI scores $\left(\chi^{2}=0.67, \mathrm{p}=0.41\right)$. High PDI scores at 20 years were associated with structural brain alterations at 15 years. In controls, those with high PDI scores showed decreased grey matter volume in parahippocampal and middle occipital gyri and decreased white matter volume in inferior temporal gyrus and precuneus. In VPT-born individuals grey matter volume decreases were observed in those with high PDI scores in superior/medial frontal and middle temporal gyri and white matter volume decreases in insula.

Conclusions High PDI scores in early adulthood are associated with region-specific structural brain alterations in mid-adolescence. Fronto-temporal alterations observed in the VPT group may reflect the neurodevelopmental vulnerability of this network, which has been implicated in the pathophysiology of delusions in psychosis.

\section{NEURODEVELOPMENTAL DISABILITIES AND MENTAL HEALTH IN EXTREME PRETERM CHILDREN. A NATIONAL POPULATION BASED STUDY}

doi:10.1136/archdischild-2012-302724.1251

${ }^{1} \mathrm{SK}$ Elgen, ${ }^{1,2} \mathrm{KT}$ Leversen, ${ }^{3} \mathrm{JH}$ Grundt, ${ }^{3} \mathrm{~J}$ Hurum, ${ }^{3} \mathrm{AB}$ Sundby, ${ }^{1,2} \mathrm{IB}$ Elgen, 1,2,3T Markestad. 'Department of Clinical Medicine, Pediatrics, University of Bergen; ${ }^{2}$ Department of Pediatrics, Haukeland University Hospital, Bergen; ${ }^{3}$ Department of Pediatrics, Hospital Innlandet Trust, Lillehammer, Norway

Objective To compare mental health at 5 years in children born extremely preterm with a reference group, and assess associations between neurodevelopmental disabilities and mental health within the preterm group.

Design In a national Norwegian cohort with gestational age (GA) 22-27 weeks or birthweight 500-999g mental health was assessed with The Strengths and Difficulties Questionnaire (SDQ), cognitive function with the Wechsler Preschool and Primary Scale of Intelligence-Revised (WPPSI-R), motor function with the Movement Assessment Battery for children (ABC-test) and severity of cerebral palsy (CP) with the Gross Motor Function Classification for CP (GMFCS). Neurodevelopmental disabilities (NDD) were described as mild and moderate/severe. SDO of the preterm children was compared with that of an unselected reference group. SDQ subscores $\geq 90^{\text {th }}$ percentile of the reference group were defined as a mental health problem and a Total Difficulties Score $\geq 90^{\text {th }}$ percentile (TDS90) as suggestive of psychiatric disorder.

Results Of 372 eligible preterm children parents completed SDO for 255 (69\%). 97(38\%) had TDS90 compared to $116(11 \%)$ of the 
reference group (OR: 5.1; 95\% CI 3.7 to 7.1). For the preterms the rate of TDS90 was higher for those with moderate/severe NDD (27/37 vs. 27/116, adjusted OR: 8.09 ; 95\% CI 3.2-19, and mild NDD 43/102 (adjusted OR: 2.2 (1.2-4.1). For preterms with no NDD TDS90 was more common than for the reference group (27/116 vs. $116 / 1089$, OR: 2.5 ; $95 \%$ CI 1.6 to 4.1 ).

Conclusion Extreme prematurity was associated with increased risk of later mental health problems, particularly if they had other neurodevelopmental disabilities.

\section{EVALUATION OF PREMATURE CHILDREN BY VOLUMETRIC MAGNETIC RESONANCE IMAGING AND COGNITIVE TESTS}

doi:10.1136/archdischild-2012-302724.1252

${ }^{1} E$ Arhan, ${ }^{2} \mathrm{~K}$ Gücüyener, ${ }^{1} \mathrm{ASS}$ Soysal, ${ }^{3} \mathrm{MA}$ Gürses, ${ }^{4} E$ Ergenekon, ${ }^{3} S ̧$ Şalvarll, ${ }^{4} \mathrm{C}$ Turkylmaz, ${ }^{4} \mathrm{E}$ Koc, ${ }^{4} \mathrm{EE}$ Onal, ${ }^{\prime} \mathrm{E}$ Demir, ${ }^{1} \mathrm{~A}$ Serdaroglu, ${ }^{4} \mathrm{Y}$ Atalay. ${ }^{1}$ Pediatric Neurology, Gazi University Faculty of Medicine; 'Pediatric Neurology, Gazi University Faculty of Medicine University; ${ }^{3}$ Radiology, INTEGRA Imaging Center; ${ }^{4}$ Neonatology, Gazi University Faculty of Medicine, Ankara, Turkey

Preterm children experience a high prevalence of long-term serious cognitive defects. Fetuses of 23 weeks of gestational age are now viable. Subsequent physiological stress can seriously disrupt the maturational processes that lay down this arthitecture. The ensuing abnormalities in brain may then contribute to the long term cogntive deficits. We aimed to measure regional brain volumes on the magnetic resonance imaging of prematurely born 9-year-old children and group matched term children. Nineteen nine year old preterm children and 21 term children recruited for the study. All subjectswent under the volumetric magnetic resonance imaging. In the neurocognitive assessment, it was observed that preterm children had impairments in visuospatial functioning, three- dimensional thought ability, data processing andlearning speed, executive function, complicated executive attention, perseveration, working memory, abstract thinking, installation replacement, focused attention. Volumes of serebellum, right and left caudate nucleus, right and left putamen, right and left globus pallidus. Right and left hypocampus and corpus callosum were significantly smaller in preterm children. There was a correlation between serebellar volume and executivefunction, harmony in social life, importance given to attentionand detail, visuospatial disorder and verbal IO, hypocampal volume and attention, arithmetics, verbal and performance IO, bilateral caudate nucleus volume and full scale IO score, vocabulary and speech skills, time in event processing, three dimensional thinking and verbal IO scores.

Our data indicate that preterm birth is associated with regionally specific, long term reductions in brain volume, in turn lead to poorer cognitive outcome.

\section{FA-VALUES IN THE PLIC AT TERM EQUIVALENT AGE ARE ASSOCIATED WITH NEURODEVELOPMENTAL OUTCOME AT TWO YEARS CORRECTED AGE}

doi:10.1136/archdischild-2012-302724.1253

K Kersbergen, N Wagenaar, B Van Kooij, F Groenendaal, LS de Vries, M Benders. Neonatology, Wilhelmina Children's Hospital/UMC, Utrecht, The Netherlands

Microstructural changes in white matter (WM) can be detected by Diffusion Tensor Imaging (DTI). This study tests the hypothesis that Fractional Anisotropy (FA) in the posterior limb of the internal capsule (PLIC), as measured directly on the FA-maps in preterm infants ATE is correlated to neuro-developmental outcome at two years corrected age.

Methods MRI at term was performed in 66 preterm infants (GA< 32 wks), Using colour-coded DTI maps, FA-values were assessed in the PLIC. Regions of interest (ROI's) were manually positioned in the PLIC bilaterally at the level of the foramen of Monro. FA-values from these ROI's were assessed in relation to scores for cognition, fine and gross motor outcome measured with the Bayley Scales of Infant and Toddler Development (BSITD)-III. Correlations were corrected for clinical factors.

Results FA-values in the left PLIC were correlated with cognition $(\mathrm{r}=0,435, \mathrm{p}<0.05)$, fine motor $(\mathrm{r}=0,335, \mathrm{p}<0.05)$ and gross motor $(r=0,337, p<0.05)$ outcome at two years corrected age. There was no such association for the right PLIC. After correction for gender, the correlation persisted only in boys. Postmenstrual age (PMA) and WMI were both significantly correlated with FA values in the left PLIC (resp $r=0.253, p<0.05$ and $r=-0.368, p<0.05$ ).

Conclusion FA-values measured by ROI-analysis ATE are significantly correlated with neurodevelopmental outcome at two years. This is in agreement with studies that used advanced postprocessing techniques to measure diffusion parameters. These measurements in this study are easy to perform, reproducible and directly applicable on MRI data and therefore particularly eligible for use in clinical care.

\section{LANGUAGE DEVELOPMENT OF PREMATURELY BORN CHILDREN - BIOLOGICAL DETERMINANTS AND SYNTAX}

doi:10.1136/archdischild-2012-302724.1254

'Z Kolundžić, ' ${ }^{2} \mathrm{M}$ Lenček, 'A Šimić Klarić. 'General Hospital, Požega; ${ }^{2}$ Faculty of Education and Rehabilitation Sciences, University of Zagreb, Zagreb, Croatia

There are numerous findings of the literature about premature born children having a higher degree of risk for different types of communication, language, reading and writing difficulties, compared to children born at term. These problems may seem minor in early language development and they are generally not recognized in that period. As the rule of language grows, particularly through reading and writing abilities, the problems become more evident and recognizable, and can significantly affect the academic achievement of children, especially if they do not provide help and support.

These theoretical findings were the basis for setting the aim of the study: to compare the outcome of prematurely borns in the age of 10 years, with the outcome of children born at term on linguistic tasks, especially in syntactic knowledge. According to this, one of the goals of the study was to investigate the relationship between biological variables (gestational age, birth weight, Apgar score) and linguistic- syntactic knowledge. The characteristics of this relationship were also examined. 34 prematurely born children and 34 children born at term participated.

Results showed statistically significant differences between prematurely born children and children born at term on syntactic knowledge tasks: prematurely borns' performings were significantly poorer. Biological factors were predictive for the level of syntactic knowledge. The purpose of this paper is to emphasize the importance of systematic monitoring of communication, language and speech development in prematurely born children.

\section{FUNCTIONAL ALTERATIONS OF THE HIPPOCAMPUS IN IUGR}

doi:10.1136/archdischild-2012-302724.1255

'M Camprubí Camprubí, 'JA Ortega Cano, 'C Duran Fernandez-Feijoo, 'I Iglesias Platas, ${ }^{3} \mathrm{~A}$ Ortega, ${ }^{1} \mathrm{X}$ Krauel, ${ }^{2} \mathrm{~S}$ Alcantara. ${ }^{1}$ Neonatology, Hospital Sant Joan de Déu, Universitat de Barcelona; '2Department of Experimental Pathology and Therapeutics, School of Medicine, University of Barcelona; ${ }^{3}$ Departamento de Celulas Troncales, CABIMER, Universidad Pablo de Olavide, Barcelona, Spain

Introduction Intrauterine growth restriction (IUGR) has been shown to relate to later neurodevelopmental problems. Recent studies suggest that deficits in spatial memory are the most prevalent among these individuals. The hippocampus, a key structure in spatial orientation, is susceptible to hypoxia or stress during pregnancy, 